\title{
THE TIME OF ULTIMATE RECOVERY IN GAUSSIAN RISK MODEL
}

\author{
KRZYSZTOF DȨBICKI AND PENG LIU
}

Abstract: We analyze the distance $\mathcal{R}_{T}(u)$ between the first and the last passage time of $\{X(t)-c t$ : $t \in[0, T]\}$ at level $u$ in time horizon $T \in(0, \infty]$, where $X$ is a centered Gaussian process with stationary increments and $c \in \mathbb{R}$, given that the first passage time occurred before $T$. Under some tractable assumptions on $X$, we find $\Delta(u)$ and $G(x)$ such that

$$
\lim _{u \rightarrow \infty} \mathbb{P}\left(\mathcal{R}_{T}(u)>\Delta(u) x\right)=G(x),
$$

for $x \geq 0$. We distinguish two scenarios: $T<\infty$ and $T=\infty$, that lead to qualitatively different asymptotics. The obtained results provide exact asymptotics of the ultimate recovery time after the ruin in Gaussian risk model.

Key Words: Gaussian risk process; exact asymptotics; first ruin time; last ruin time; generalized Pickands-Piterbarg constant.

AMS Classification: Primary 60G15; secondary 60G70,60K25

\section{INTRODUCTION}

For given threshold $u>0$ and time horizon $T \in(0, \infty]$, let

$$
\tau_{T}(u):=\inf \{t \geq 0: X(t)-c t>u, 0 \leq t \leq T\}
$$

and

$$
\mathcal{T}_{T}(u):=\sup \{t \geq 0: X(s)-c s>u, 0 \leq t \leq T\},
$$

be the first and the last passage time of process $X(t)-c t, t \geq 0$ at level $u$ respectively, with convention that $\inf \emptyset=\infty$ and $\sup \emptyset=0$.

The analysis of properties of $\tau_{T}(u)$ and $\mathcal{T}_{T}(u)$, due to their obvious importance in extreme value theory of stochastic processes, attracted substantial interest, being additionally stimulated by relations of passage times with important problems in applied probability. More specifically, in risk theory $\tau_{T}(u)$ and $\mathcal{T}_{T}(u)$ have the interpretation as the first and the last ruin time of the risk reserve process $S(t):=u+c t-X(t)$, where $u>0$ is the initial capital, $c>0$ is the premium rate and $X(t), t \geq 0$ is the accumulated claim amount in interval $(0, t]$; see e.g. [3, 18].

In this contribution we suppose that $X$ is a centered Gaussian process with stationary increments, a.s. continuous sample paths, $X(0)=0$ and $c>0$. We note that in the context of risk theory, there are strong application-based and theoretical reasons for modelling accumulated claim amount by Gaussian processes with stationary increments. On one hand the family of Gaussian processes provides flexibility in the adjustment of suitable correlation model, since it covers wide range of correlation structures. On the other hand, there are theoretical results that legitimate approximation of 
the accumulated claim amount in highly aggregated models by Gaussian processes; see the celebrated work by Iglehart [22] for the Brownian approximation and, e.g., [7, 18, 26] for more general models, including e.g. fractional Brownian motion approximations.

This contribution is devoted to analysis of the distribution of

$$
\mathcal{R}_{T}(u):=\mathcal{T}_{T}^{*}(u)-\tau_{T}^{*}(u)
$$

where

$$
\left(\tau_{T}^{*}(u), \mathcal{T}_{T}^{*}(u)\right):=\left(\tau_{T}(u), \mathcal{T}_{T}(u)\right) \mid\left(\tau_{T}(u)<\infty\right),
$$

both for $T \in(0, \infty)$ and $T=\infty$. Referring again to risk theory, $\mathcal{R}_{T}(u)$ has the interpretation as the ultimate time to recovery, which is the difference between the last and first ruin time, under the condition that ruin occurred; see also $[14,23]$ and references therein. We note that $\mathcal{R}_{T}(u)$ is also closely related to the so-called Parisian ruin time, which is the first time that the length of the consecutive excursion period of the surplus process $S$ under level 0 exceeds a pre-specified time threshold; see, e.g., [2], [25], [4] and [5], with straightforward observation that $\mathcal{R}_{T}(u)$ gives an upper bound for the appropriately chosen pre-specified time period in Parisian model. Another related notion is the cumulative ruin time, which is based on the total time spent below 0 (in red) by the underlying risk process; see, e.g., [15]. Clearly, $\mathcal{R}_{T}(u)$ is greater than the corresponding occupation time.

For $T=\infty$, the asymptotics of the distribution of conditional first and last ruin times in Gaussian risk context were studied in, e.g., [20] and [21]; see also [18], [24] and [7] for related $\gamma$-reflected Gaussian models. Specifically, under some tractable assumptions on $X$, the following asymptotics was found in $[7,20,21]$ :

$$
\left(\frac{\tau_{\infty}^{*}(u)-u t_{u}}{\sigma\left(u t_{u}\right)}, \frac{\mathcal{T}_{\infty}^{*}(u)-u t_{u}}{\sigma\left(u t_{u}\right)}\right) \stackrel{d}{\rightarrow}(\mathrm{C} \mathcal{N}, \mathrm{C} \mathcal{N}), \quad u \rightarrow \infty
$$

where $\sigma^{2}(t)=\operatorname{Var}(X(t)), t_{u}=\arg _{\inf _{t>0}} \frac{u(1+c t)}{\sigma(u t)}, \mathcal{N} \sim N(0,1)$ and $\mathrm{C}$ is some known constant. However, the above result is too crude in order to deal with $\mathcal{R}_{\infty}(u)$, since it follows straightforwardly from (2), that

$$
\frac{\mathcal{T}_{\infty}^{*}(u)-\tau_{\infty}^{*}(u)}{\sigma\left(u t_{u}\right)} \rightarrow 0, \quad u \rightarrow \infty
$$

in probability; see also Corollary 4 in [21]. This implies that $\sigma\left(u t_{u}\right)$ acting as a scaling function is too big, so in order to get a nontrivial result one has to scale by asymptotically smaller function.

The main results of this contribution provide $\Delta(u)$ and distribution function $G(\cdot)$ such that

$$
\lim _{u \rightarrow \infty} \mathbb{P}\left(\mathcal{R}_{T}(u)>x \Delta(u)\right)=G(x),
$$

for $x \geq 0$, both for $T \in(0, \infty)$ and $T=\infty$.

As it is shown in Theorems 2.1, 2.3, both $\Delta(u)$ and $G(x)$ depend on $T$ and on the local behavior of variance function of $X$, which leads to several scenarios. Interestingly, the limit function $G$ is given in terms of generalized Pickands-Piterebarg-type constants. In order to obtain the main results of this contribution we accommodate to our needs recently developed uniform double-sum method applied for relevant continuous functionals; see [6]. 
Organization of the paper: Section 2 is devoted to introduction of notation and presentation of main results. In section 3 we present proofs of the main results.

\section{MAIN RESUlts}

In this section we provide main results of this contribution, which is the limit theorem for

$$
\mathcal{R}_{T}(u)=\mathcal{T}_{T}^{*}(u)-\tau_{T}^{*}(u)
$$

as $u \rightarrow \infty$, for $T \in(0, \infty]$. Due to specific asymptotic nature of $\mathcal{R}_{T}(u)$ we distinguish two separate scenarios: infinite-time horizon $(T=\infty)$ and finite-time horizon $(T<\infty)$.

2.1. Infinite-time horizon. Suppose that $T=\infty$. Consider a centered Gaussian process $X$ with continuous trajectories, stationary increments and variance function $\sigma^{2}(t):=\operatorname{Var}(X(t))$ such that AI: $\sigma^{2}(0)=0, \sigma^{2}(t)$ is regularly varying at $\infty$ with index $2 \alpha_{\infty} \in(0,2)$ and $\sigma^{2}(t)$ is twice continuously differentiable on $(0, \infty)$ with its first derivative $\dot{\sigma}^{2}(t):=\frac{\mathrm{d} \sigma^{2}}{\mathrm{~d} t}(t)$ and second derivative $\ddot{\sigma^{2}}(t):=\frac{\mathrm{d}^{2} \sigma^{2}}{\mathrm{~d} t^{2}}(t)$ being ultimately monotone at $\infty$.

AII: $\sigma^{2}(t)$ is regularly varying at 0 with index $2 \alpha_{0} \in(0,2]$.

For given $x \geq 0$ and $f \in C([0,2 S]), S>0$, let

$$
\Gamma(x, S ; f)=\sup _{t \in[0, S]} \min \left(f(t), \sup _{s \in[t+x, 2 S]} f(s)\right) .
$$

Then, for given $\eta(t), t \geq 0$, a centered Gaussian process with stationary increments and continuous sample paths, we introduce

$$
\begin{aligned}
\mathcal{H}_{\eta}^{\Gamma}(x, S): & =\mathbb{E}(\exp (\Gamma(x, S ; \sqrt{2} \eta(t)-\operatorname{Var}(\eta(t))))), \\
\mathcal{H}_{\eta}^{\Gamma}(x): & =\lim _{S \rightarrow \infty} \frac{\mathcal{H}_{\eta}^{\Gamma}(x, S)}{S}
\end{aligned}
$$

providing that the limit exists. We note that $\mathcal{H}_{\eta}^{\Gamma}(0)$ coincides with the notion of generalized Pickands constant, since

$$
\mathcal{H}_{\eta}^{\Gamma}(0, S)=\mathbb{E}\left(\exp \left(\sup _{t \in[0, S]}(\sqrt{2} \eta(t)-\operatorname{Var}(\eta(t)))\right)\right) ;
$$

see [27], [10], [19], [11] [8], [16], [13], [12] and [17] for properties of generalized Pickands constants. In order to simplify notation, let

$$
\mathcal{H}_{\eta}:=\mathcal{H}_{\eta}^{\Gamma}(0), \quad \text { and } \quad \mathcal{H}_{\eta}([0, S]):=\mathcal{H}_{\eta}^{\Gamma}(0, S)
$$

Let $B_{H}(t)$ denote the standard fractional Brownian motion with mean 0 and correlation function satisfying

$$
\operatorname{Cov}\left(B_{H}(s), B_{H}(t)\right)=\frac{|s|^{2 H}+|t|^{2 H}-|t-s|^{2 H}}{2}, \quad s, t \geq 0, H \in(0,1] .
$$

Let $t^{*}=\frac{\alpha_{\infty}}{c\left(1-\alpha_{\infty}\right)}$ and $\overleftarrow{\sigma}(t), t \geq 0$ stand for the asymptotic inverse function of $\sigma$ at value of $t$ Furthermore, let

$$
\Delta(u)=\overleftarrow{\sigma}\left(\frac{\sqrt{2} \sigma^{2}\left(u t^{*}\right)}{u\left(1+c t^{*}\right)}\right)
$$


In the rest of this section we tacitly assume that

$$
\varphi:=\lim _{u \rightarrow \infty} \frac{\sigma^{2}(u)}{u} \in[0, \infty] .
$$

Theorem 2.1. Let $X(t)$ be a centered Gaussian process with continuous trajectories and stationary increments satisfying AI-AII. Then for any $x \geq 0$

$$
\lim _{u \rightarrow \infty} \mathbb{P}\left(\frac{\mathcal{R}_{\infty}(u)}{\Delta(u)}>x\right)=\frac{\mathcal{H}_{\eta}^{\Gamma}(x)}{\mathcal{H}_{\eta}^{\Gamma}(0)} \in(0,1],
$$

where

$$
\eta(t)=\left\{\begin{array}{cc}
B_{\alpha_{0}}(t), & \varphi=0 \\
\frac{X(\varphi t)}{\sigma(\varphi)}, & \varphi \in(0, \infty) \\
B_{\alpha_{\infty}}(t), & \varphi=\infty
\end{array}\right.
$$

Remark 2.2. Due to the covariance decomposition in Lemma 3.2, process $\eta(t)$ in the generalized Pickands-Piterbarg constant is determined by $\lim _{u \rightarrow \infty} \frac{\sigma^{2}(\Delta(u) t)}{\sigma^{2}(\Delta(u))}$. In particular, if $\varphi=0$, then $\lim _{u \rightarrow \infty} \Delta(u)=0$ and thus, by the regularity of $\sigma^{2}(t)$ at 0 , it follows that $\lim _{u \rightarrow \infty} \frac{\sigma^{2}(\Delta(u) t)}{\sigma^{2}(\Delta(u))}=t^{2 \alpha_{0}}$, which is the variance of fractional Brownian motion $B_{2 \alpha_{0}}(t)$. The case $\varphi=\infty$ leads to $B_{2 \alpha_{\infty}}(t)$ by the same argument. If $\varphi \in(0, \infty)$, then $\lim _{u \rightarrow \infty} \Delta(u) \in(0, \infty)$, implying that $\lim _{u \rightarrow \infty} \frac{\sigma^{2}(\Delta(u) t)}{\sigma^{2}(\Delta(u))}=\frac{\sigma^{2}(\varphi t)}{\sigma^{2}(\varphi)}$, which corresponds to the variance of $\frac{X(\varphi t)}{\sigma(\varphi)}$.

2.2. Finite-time horizon. In this subsection we focus on the finite-time case, i.e. we suppose that $T \in(0, \infty)$. Let $X(t), t \in[0, T]$ be a Gaussian process with stationary increments, a.s. continuous trajectories, zero-mean and variance function $\sigma^{2}$ satisfying

BI $\sigma^{2}(0)=0$ and $\sigma^{2} \in C(0, T]$ with the first derivative being positive.

BII $\sigma^{2}$ is regularly varying at 0 with index $2 \alpha_{0} \in(0,2]$.

Denote by

$$
\Delta_{1}(u)=\overleftarrow{\sigma}\left(\frac{\sqrt{2} \sigma^{2}(T)}{u+c T}\right), \quad \Delta_{2}(u)=\left(\frac{\sigma(T)}{u+c T}\right)^{2}
$$

and

$$
\begin{aligned}
\mathcal{P}_{B_{1 / 2}}^{d}(x)= & \int_{0}^{\infty} e^{w} \mathbb{P}\left(\sup _{t \in[x, \infty)} \min \left(\sup _{s \in[0, t-x]} \sqrt{2} B_{1 / 2}(s)-(1+d) s, \sqrt{2} B_{1 / 2}(t)-(1+d) t\right)>w\right) d w \\
& +\int_{-\infty}^{0} e^{w} \mathbb{P}\left(\sup _{t \in[x, \infty)}\left(\sqrt{2} B_{1 / 2}(t)-(1+d) t\right)>w\right) d w
\end{aligned}
$$

with $B_{1 / 2}$ a standard Brownian motion and $d>0$. We note that

$$
\mathcal{P}_{B_{1 / 2}}^{d}(0)=\mathbb{E}\left(\exp \left(\sup _{t \in[0, \infty)}\left(\sqrt{2} B_{1 / 2}(t)-(1+d) t\right)\right)\right),
$$

is the classical Piterbarg constant (see [1] and reference therein) and hence

$$
\mathcal{P}_{B_{1 / 2}}^{d}(0)=1+\frac{1}{d}
$$

see, e.g., [27]. 
Theorem 2.3. Let $X(t), t \in[0, T]$ be a centered Gaussian process with continuous trajectories and stationary increments satisfying BI-BII and $x \geq 0$.

i) If $t=o\left(\sigma^{2}(t)\right), t \rightarrow 0$, then

$$
\lim _{u \rightarrow \infty} \mathbb{P}\left(\frac{\mathcal{R}_{T}(u)}{\Delta_{1}(u)}>x\right)=\frac{\mathcal{H}_{B_{\alpha_{0}}}^{\Gamma}(x)}{\mathcal{H}_{B_{\alpha_{0}}}^{\Gamma}(0)} \in(0,1]
$$

ii) If $\sigma^{2}(t) \sim a t, t \rightarrow 0, a>0$, then for $d=\frac{2 \sigma(T) \dot{\sigma}(T)}{a}$,

$$
\lim _{u \rightarrow \infty} \mathbb{P}\left(\frac{\mathcal{R}_{T}(u)}{\Delta_{1}(u)}>x\right)=\frac{\mathcal{P}_{B_{1 / 2}}^{d}(x)}{\mathcal{P}_{B_{1 / 2}}^{d}(0)} \in(0,1] .
$$

iii) If $\sigma^{2}(t)=o(t), t \rightarrow 0$, then

$$
\lim _{u \rightarrow \infty} \mathbb{P}\left(\frac{\mathcal{R}_{T}(u)}{\Delta_{2}(u)}>x\right)=e^{-\frac{\dot{\sigma}(T)}{\sigma(T)} x}
$$

\section{PROOFs}

In this section, we give proofs of Theorem 2.1 and Theorem 2.3. Hereafter, denote by $\mathbb{Q}, \mathbb{Q}_{i}, i=$ $1,2,3, \ldots$ positive constants that may differ from line to line and $\bar{X}:=\frac{X}{\sqrt{\operatorname{Var}(X)}}$ for any nontrivial random variable $X$. Moreover, $f(u) \sim g(u), u \rightarrow \infty$ means that $\lim _{u \rightarrow \infty} \frac{f(u)}{g(u)}=1$. In our proofs, multiple limits appear. We shall write for instance

$$
b(u, S, \epsilon) \sim a(u), \quad u \rightarrow \infty, S \rightarrow \infty, \epsilon \rightarrow 0
$$

to mean that

$$
\lim _{\epsilon \rightarrow 0} \lim _{S \rightarrow \infty} \lim _{u \rightarrow \infty} \frac{b(u, S, \epsilon)}{a(u)}=1 .
$$

3.1. Infinite-time horizon. Observe that for any $x \geq 0$

$$
\mathbb{P}\left(\frac{\mathcal{T}_{\infty}^{*}(u)-\tau_{\infty}^{*}(u)}{\Delta(u)}>x\right)=\frac{\mathbb{P}\left(\mathcal{T}_{\infty}(u)-\tau_{\infty}(u)>x \Delta(u), \tau_{\infty}<\infty\right)}{\mathbb{P}\left(\tau_{\infty}(u)<\infty\right)},
$$

with $\Delta(u)$ defined in (6). In order to derive the limiting distribution of the above ratio, we need to derive the asymptotics of $\mathbb{P}\left(\mathcal{T}_{\infty}(u)-\tau_{\infty}(u)>x \Delta(u), \tau_{\infty}<\infty\right)$ and $\mathbb{P}\left(\tau_{\infty}(u)<\infty\right)$ respectively. Using that

$$
\mathbb{P}\left(\tau_{\infty}(u)<\infty\right)=\mathbb{P}\left(\sup _{t \geq 0} X(t)-c t>u\right)=\mathbb{P}\left(\sup _{t \geq 0} X_{u}(t)>m(u)\right),
$$

where $X_{u}(t)=\frac{X(u t)}{u(1+c t)} m(u)$ with $m(u)=\inf _{t>0} \frac{u(1+c t)}{\sigma(u t)}$, Proposition 2 in [11] (or Theorems 3.1-3.3 in [9]), implies the following Lemma.

Lemma 3.1. Let $X(t)$ be a centered Gaussian process with continuous trajectories and stationary increments satisfying AI-AII. Assuming that $\lim _{u \rightarrow \infty} \frac{\sigma^{2}(u)}{u} \in[0, \infty]$, we have

$$
\mathbb{P}\left(\tau_{\infty}(u)<\infty\right) \sim \mathcal{H}_{\eta}^{\Gamma}(0) \sqrt{\frac{2 A \pi}{B}} \frac{u}{m(u) \Delta(u)} \Psi(m(u)),
$$

where $\Delta(u)$ is defined in (6), $\eta$ is defined in (7) and

$$
A=\left(\frac{\alpha_{\infty}}{c\left(1-\alpha_{\infty}\right)}\right)^{-\alpha_{\infty}} \frac{1}{1-\alpha_{\infty}}, \quad B=\left(\frac{\alpha_{\infty}}{c\left(1-\alpha_{\infty}\right)}\right)^{-\alpha_{\infty}-2} \alpha_{\infty} .
$$


Thus, by (9), we are left with finding the asymptotics of $\mathbb{P}\left(\mathcal{T}_{\infty}(u)-\tau_{\infty}(u)>x \Delta(u), \tau_{\infty}<\infty\right)$, as $u \rightarrow \infty$.

In the next lemma we focus on asymptotic properties of the variance and correlation functions of relevant Gaussian processes; we refer to, e.g., [9] for the proof. Let

$$
\sigma_{u}(t):=\operatorname{Var}^{1 / 2}\left(X_{u}(t)\right)=\operatorname{Var}^{1 / 2}\left(\frac{X(u t)}{u(1+c t)} m(u)\right), t \geq 0, \quad t_{u}=\arg \inf _{t>0} \frac{u(1+c t)}{\sigma(u t)} .
$$

Lemma 3.2. Suppose that AI-AII are satisfied. For $u$ large enough $t_{u}$ is unique, and $t_{u} \rightarrow t^{*}=$ $\frac{\alpha_{\infty}}{c\left(1-\alpha_{\infty}\right)}$, as $u \rightarrow \infty$. Moreover, for any $\delta_{u}>0$ with $\lim _{u \rightarrow \infty} \delta_{u}=0$

$$
\lim _{u \rightarrow \infty} \sup _{t \in\left(t_{u}-\delta_{u}, t_{u}+\delta_{u}\right) \backslash\left\{t_{u}\right\}}\left|\frac{1-\sigma_{u}(t)}{\frac{B}{2 A}\left(t-t_{u}\right)^{2}}-1\right|=0,
$$

and

$$
\lim _{u \rightarrow \infty} \sup _{s \neq t, s, t \in\left(t_{u}-\delta_{u}, t_{u}+\delta_{u}\right)}\left|\frac{1-\operatorname{Cor}(X(u s), X(u t))}{\frac{\sigma^{2}(u|s-t|)}{2 \sigma^{2}\left(u t^{*}\right)}}-1\right|=0 .
$$

Proof of Theorem 2.1 Due to (9) and Lemma 3.1, we focus on the asymptotics of

$$
\begin{aligned}
\mathbb{P} & \left(\mathcal{T}_{\infty}(u)-\tau_{\infty}(u)>x \Delta(u), \tau_{\infty}<\infty\right) \\
& =\mathbb{P}(\exists s, t \geq 0, s-t \geq \Delta(u) x, X(t)-c t>u, X(s)-c s>u),
\end{aligned}
$$

as $u \rightarrow \infty$, for any $x \geq 0$. We have

$$
\pi_{1}(u) \leq \mathbb{P}\left(\mathcal{T}_{\infty}(u)-\tau_{\infty}(u)>x \Delta(u), \tau_{\infty}<\infty\right) \leq \pi_{1}(u)+\pi_{2}(u)
$$

where

$$
\begin{aligned}
& \pi_{1}(u)=\mathbb{P}\left(\exists s, t \in E_{1}(u), s-t \geq \Delta(u) x, X(t)-c t>u, X(s)-c s>u\right) \\
& \pi_{2}(u)=\mathbb{P}\left(\exists(s, t) \in[0, \infty)^{2} \backslash E_{1}^{2}(u), s-t \geq \Delta(u) x, X(t)-c t>u, X(s)-c s>u\right),
\end{aligned}
$$

with

It follows that for $u>0$

$$
E_{1}(u)=\left[u t_{u}-\frac{u \ln m(u)}{m(u)}, u t_{u}+\frac{u \ln m(u)}{m(u)}\right]
$$

$$
\pi_{2}(u) \leq 2 \mathbb{P}\left(\sup _{t \in[0, \infty) \backslash E_{1}(u)} X(t)-c t>u\right) .
$$

Hence, following Lemma 7 in [11] (or Lemma 5.6 in [9]), we have that

$$
\pi_{2}(u) \leq 2 \mathbb{P}\left(\sup _{t \in[0, \infty) \backslash E_{1}(u)} X(t)-c t>u\right)=o\left(\mathbb{P}\left(\tau_{\infty}(u)<\infty\right)\right), \quad u \rightarrow \infty .
$$

Thus we are left with finding the exact asymptotics of $\pi_{1}(u)$ as $u \rightarrow \infty$. Replacing $t$ by $u t_{u}+\Delta(u) t$ and $s$ by $u t_{u}+\Delta(u) s$, we rewrite

$$
\pi_{1}(u)=\mathbb{P}\left(\exists s, t \in E_{2}(u), s-t \geq x, Z_{u}(t)>m(u), Z_{u}(s)>m(u)\right)
$$

with

$$
Z_{u}(t)=\frac{X\left(u t_{u}+\Delta(u) t\right)}{u\left(1+c t_{u}\right)+c \Delta(u) t} m(u), \quad E_{2}(u)=\left[-\frac{u \ln m(u)}{\Delta(u) m(u)}, \frac{u \ln m(u)}{\Delta(u) m(u)}\right]
$$


Bonferroni inequality gives that for $S>x$,

$$
\Sigma_{1}^{+}(u)-\Sigma \Sigma_{1}(u) \leq \pi_{1}(u) \leq \Sigma_{1}^{-}(u)+\Sigma \Sigma_{2}(u)
$$

where

$$
\begin{aligned}
\Sigma_{1}^{ \pm}(u) & =\sum_{k=-N(u) \pm 1}^{N(u) \mp 1} \mathbb{P}\left(\exists t \in[k S,(k+1) S], s \in[t+x,(k+2) S]: \min \left(Z_{u}(t), Z_{u}(s)\right)>m(u)\right) \\
\Sigma \Sigma_{1}(u) & =\sum_{k=-N(u)-1}^{N(u)+1} \sum_{l=k+1}^{N(u)+1} \mathbb{P}\left(\sup _{t \in[k S,(k+1) S]} Z_{u}(t)>m(u), \sup _{t \in[l S,(l+1) S]} Z_{u}(t)>m(u)\right) \\
\Sigma \Sigma_{2}(u) & =\sum_{k=-N(u)-1}^{N(u)+1} \sum_{l=k+2}^{N(u)+1} \mathbb{P}\left(\sup _{t \in[k S,(k+1) S]} Z_{u}(t)>m(u), \sup _{t \in[l S,(l+1) S]} Z_{u}(t)>m(u)\right)
\end{aligned}
$$

where $N(u)=\left[\frac{u \ln m(u)}{\Delta(u) m(u) S}\right]$. To get the asymptotics of $\pi_{1}(u)$, in next steps of the proof we show that $\Sigma_{1}^{+}(u) \sim \Sigma_{1}^{-}(u)$ and $\Sigma \Sigma_{2}(u) \leq \Sigma \Sigma_{1}(u)=o\left(\Sigma_{1}^{+}(u)\right)$, as $u \rightarrow \infty$ and $S \rightarrow \infty$.

Asymptotics of $\Sigma_{1}^{ \pm}(u)$. Setting

$$
Z_{u, k}(t)=Z_{u}(k S+t)
$$

we have that

$$
\begin{aligned}
\Sigma_{1}^{-}(u) & =\sum_{k=-N(u)-1}^{N(u)+1} \mathbb{P}\left(\sup _{t \in[k S,(k+1) S]} \min \left(Z_{u}(t), \sup _{s \in[t+x,(k+2) S]} Z_{u}(s)\right)>m(u)\right) \\
& =\sum_{k=-N(u)-1}^{N(u)+1} \mathbb{P}\left(\Gamma\left(x, S ; Z_{u, k}\right)>m(u)\right) \\
& \leq \sum_{k=-N(u)-1}^{N(u)+1} \mathbb{P}\left(\Gamma\left(x, S ; \bar{Z}_{u, k}\right)>\frac{m(u)}{\sup _{t \in[0,2 S]} \sqrt{\operatorname{Var}\left(Z_{u, k}(t)\right)}}\right),
\end{aligned}
$$

where $\Gamma$ is defined in (3). By Lemma 3.2, for any $0<\epsilon<1$

$$
\frac{m(u)}{\sup _{t \in[k S,(k+1) S]} \sqrt{\operatorname{Var}\left(Z_{u, k}(t)\right)}} \geq m(u)\left(1+(1-\epsilon) \frac{B}{2 A}\left(|k|^{*} \frac{\Delta(u)}{u} S\right)^{2}\right):=m_{k, \epsilon}(u)
$$

with $|k|^{*}=\min (|k|,|k+1|,|k+2|)$ as $u$ sufficiently large. Thus for $0<\epsilon<1$ and $u$ sufficiently large

$$
\Sigma_{1}^{-}(u) \leq \sum_{k=-N(u)-1}^{N(u)+1} \mathbb{P}\left(\Gamma\left(x, S ; \bar{Z}_{u, k}\right)>m_{k, \epsilon}(u)\right)
$$

Using that

$$
\lim _{u \rightarrow \infty} \sup _{|k| \leq N(u)+1}\left|\frac{2 \sigma^{2}\left(u t^{*}\right)}{\sigma^{2}(\Delta(u))\left(m_{k, \epsilon}(u)\right)^{2}}-1\right|=0
$$

by Lemma 3.2

$$
\lim _{u \rightarrow \infty} \sup _{|k| \leq N(u)+1} \sup _{s \neq t, s, t \in[0,2 S]}\left|m_{k, \epsilon}^{2}(u) \frac{1-\operatorname{Cor}\left(Z_{u, k}(s), Z_{u, k}(t)\right)}{\frac{\sigma^{2}(\Delta(u)|s-t|)}{\sigma^{2}(\Delta(u))}}-1\right|=0 .
$$


Hence in light of Proposition 2.3 in [6],

$$
\lim _{u \rightarrow \infty} \sup _{|k| \leq N(u)+1}\left|\frac{\mathbb{P}\left(\Gamma\left(x, S ; \bar{Z}_{u, k}(t)\right)>m_{k, \epsilon}(u)\right)}{\Psi\left(m_{k, \epsilon}(u)\right)}-\mathcal{H}_{\eta}^{\Gamma}(x, S)\right|=0,
$$

where $\eta$ is defined in (7). Furthermore,

$$
\begin{aligned}
\Sigma_{1}^{-}(u) & \leq \sum_{k=-N(u)-1}^{N(u)+1} \mathcal{H}_{\eta}^{\Gamma}(x, S) \Psi\left(m_{k, \epsilon}(u)\right) \\
& \sim \mathcal{H}_{\eta}^{\Gamma}(x, S) \Psi(m(u)) \sum_{k=-N(u)-1}^{N(u)+1} e^{-(1-\epsilon) \frac{B}{2 A} m^{2}(u)\left(|k|^{*} \frac{\Delta(u)}{u} S\right)^{2}} \\
& \sim \frac{\mathcal{H}_{\eta}^{\Gamma}(x, S)}{S} \frac{(1-\epsilon)^{-1 / 2}\left(\frac{B}{2 A}\right)^{-1 / 2} u}{m(u) \Delta(u)} \Psi(m(u)) \int_{-\infty}^{\infty} e^{-t^{2}} d t \\
& \sim \frac{\mathcal{H}_{\eta}^{\Gamma}(x, S)}{S} \Theta(u), \quad u \rightarrow \infty, \epsilon \rightarrow 0
\end{aligned}
$$

where

$$
\Theta(u):=\sqrt{\frac{2 A \pi}{B}} \frac{u}{m(u) \Delta(u)} \Psi(m(u))
$$

Similarly,

$$
\Sigma_{1}^{+}(u) \geq \frac{\mathcal{H}_{\eta}^{\Gamma}(x, S)}{S} \Theta(u)(1+o(1)), \quad u \rightarrow \infty .
$$

Upper bound for $\Sigma \Sigma_{i}(u), i=1,2$. Similarly as in (14), Lemma 3.2 gives that

$$
\lim _{u \rightarrow \infty} \sup _{s \neq t, s, t \in E_{2}(u)}\left|m^{2}(u) \frac{1-\operatorname{Cor}\left(Z_{u}(s), Z_{u}(t)\right)}{\frac{\sigma^{2}(\Delta(u)|s-t|)}{\sigma^{2}(\Delta(u))}}-1\right|=0
$$

By Corollary 3.2 in [6], there exists $\mathcal{C}, \mathcal{C}_{1}>0$ such that for all $|k|,|l| \leq N(u)+1, l \geq k+2$,

$$
\mathbb{P}\left(\sup _{t \in[k S,(k+1) S]} \overline{Z_{u}}(t)>m_{k, \epsilon}(u), \sup _{t \in[l S,(l+1) S]} \overline{Z_{u}}(t)>m_{l, \epsilon}(u)\right) \leq \mathcal{C} S^{2} e^{-\mathcal{C}_{1}|k-l|^{\gamma} S^{\gamma}} \Psi\left(\hat{m}_{u, k, l}\right),
$$

with $\gamma=\min \left(\alpha_{0}, \alpha_{\infty}\right)$ and $\hat{m}_{k, l}(u)=\min \left(m_{k, \epsilon}(u), m_{l, \epsilon}(u)\right)$. Consequently, with aid of (15),

$$
\begin{aligned}
\Sigma \Sigma_{2}(u) & \leq \sum_{k=-N(u)-1} \sum_{l=k+2}^{N(u)+1} \mathcal{C} S^{2} e^{-\mathcal{C}_{1}|k-l|^{\gamma} S^{\gamma}} \Psi\left(\hat{m}_{k, l}(u)\right) \\
& \leq \sum_{k=-N(u)-1}^{N(u)+1} \sum_{l=k+2}^{N(u)+1} \mathcal{C} S^{2} e^{-\mathcal{C}_{1}|k-l|^{\gamma} S^{\gamma}}\left(\Psi\left(m_{k, \epsilon}(u)\right)+\Psi\left(m_{k, \epsilon}(u)\right)\right) \\
& \leq 2 \sum_{k=-N(u)-1}^{N(u)+1} \Psi\left(m_{k, \epsilon}(u)\right) \sum_{|k-l| \geq 1} \mathcal{C} S^{2} e^{-\mathcal{C}_{1}|k-l|^{\gamma} S^{\gamma}} \\
& \leq 2 \sum_{k=-N(u)-1}^{N(u)+1} \Psi\left(m_{k, \epsilon}(u)\right) \mathcal{C} S^{2} e^{-\mathbb{Q} S^{\gamma}} \\
& \leq \mathbb{Q} S e^{-\mathbb{Q} S^{\gamma}} \Theta(u), \quad u \rightarrow \infty .
\end{aligned}
$$


Thus, again by Proposition 2.3 in [6], taking into account (14) and noting that $\Gamma(0, S ; f)=\sup _{t \in[0, S]} f(t)$, we have

$$
\lim _{u \rightarrow \infty} \sup _{|k| \leq N(u)+1}\left|\frac{\mathbb{P}\left(\sup _{t \in[0, S]} \overline{Z_{u, k}}(t)>\hat{m}_{k, k+1}(u)\right)}{\Psi\left(\hat{m}_{k, k+1}(u)\right)}-\mathcal{H}_{\eta}([0, S])\right|=0
$$

Hence,

$$
\begin{aligned}
\hat{\Sigma}(u):= & \sum_{k=-N(u)-1}^{N(u)+1} \mathbb{P}\left(\sup _{t \in[k S,(k+1) S]} Z_{u}(t)>m(u), \sup _{t \in[(k+1) S,(k+2) S]} Z_{u}(t)>m(u)\right) \\
= & \left.\sum_{k=-N(u)-1}^{N(u)+1}\left(\mathbb{P}\left(\sup _{t \in[0, S]} \overline{Z_{u, k}}(t)>\hat{m}_{k, k+1}(u)\right)+\mathbb{P}\left(\sup _{t \in[0, S]} \overline{Z_{u, k+1}}(t)>\hat{m}_{k, k+1}(u)\right)\right)\right) \\
& -\sum_{k=-N(u)-1}^{N(u)+1} \mathbb{P}\left(\sup _{t \in[0,2 S]} \overline{Z_{u, k}}(t)>\hat{m}_{k, k+1}(u)\right) \\
\leq & \left(2 \frac{\mathcal{H}_{\eta}([0, S])}{S}-\frac{\mathcal{H}_{\eta}([0,2 S])}{S}\right) \Theta(u), \quad u \rightarrow \infty
\end{aligned}
$$

which together with (18) and the fact that

$$
\Sigma \Sigma_{1}(u)=\Sigma \Sigma_{2}(u)+\hat{\Sigma}(u)
$$

leads to

$$
\Sigma \Sigma_{1}(u) \leq\left(2 \frac{\mathcal{H}_{\eta}([0, S])}{S}-\frac{\mathcal{H}_{\eta}([0,2 S])}{S}+\mathbb{Q} S e^{-\mathbb{Q} S^{\gamma}}\right) \Theta(u), \quad u \rightarrow \infty .
$$

Combination of (13), (15), (17)-(19) yields

$$
\begin{aligned}
\liminf _{u \rightarrow \infty} \frac{\pi_{1}(u)}{\Theta(u)} & \geq \frac{\mathcal{H}_{\eta}^{\Gamma}(x, S)}{S}-2 \frac{\mathcal{H}_{\eta}([0, S])}{S}+\frac{\mathcal{H}_{\eta}([0,2 S])}{S}-\mathbb{Q} S e^{-\mathbb{Q} S^{\gamma}}, \\
\limsup _{u \rightarrow \infty} \frac{\pi_{1}(u)}{\Theta(u)} & \leq \frac{\mathcal{H}_{\eta}^{\Gamma}(x, S)}{S}+\mathbb{Q} S e^{-\mathbb{Q} S^{\gamma}} .
\end{aligned}
$$

Thus under the proviso that

$$
\mathcal{H}_{\eta}^{\Gamma}(x)=\lim _{S \rightarrow \infty} \frac{\mathcal{H}_{\eta}^{\Gamma}(x, S)}{S} \in(0, \infty),
$$

letting $S \rightarrow \infty$ in (20) leads to

$$
\lim _{u \rightarrow \infty} \frac{\pi_{1}(u)}{\Theta(u)}=\mathcal{H}_{\eta}^{\Gamma}(x) \in(0, \infty)
$$

which combined with (9)-(11) establishes the claim.

Existence of $\mathcal{H}_{\eta}^{\Gamma}(x)$. In order to complete the proof, we are left with proving (21). By (20), we have

$$
\liminf _{S \rightarrow \infty} \frac{\mathcal{H}_{\eta}^{\Gamma}(x, S)}{S}=\limsup _{S \rightarrow \infty} \frac{\mathcal{H}_{\eta}^{\Gamma}(x, S)}{S} .
$$

By the fact that

$$
\mathcal{H}_{\eta}^{\Gamma}(x, S) \leq \mathcal{H}_{\eta}([0, S])
$$

we have

$$
\limsup _{S \rightarrow \infty} \frac{\mathcal{H}_{\eta}^{\Gamma}(x, S)}{S} \leq \mathcal{H}_{\eta}<\infty
$$


In order to prove positivity of $\mathcal{H}_{\eta}^{\Gamma}(x)$, we follow the same argument as in the proof of Theorem D.2 in [27]. Replacing $\pi_{1}(u)$ in (12) by

$$
\mathbb{P}\left(\exists s \in E_{2}(u), t \in E_{2}(u) \cap \bigcup_{k \in \mathbb{Z}}[2 k S,(2 k+1) S], s-t \geq x, Z_{u}(t)>m(u), Z_{u}(s)>m(u)\right)
$$

and following the same arguments as for $\pi_{1}(u)$, we derive that for sufficiently large $\hat{S}>x$

$$
\liminf _{S \rightarrow \infty} \frac{\mathcal{H}_{\eta}^{\Gamma}(x, S)}{S} \geq \frac{\mathcal{H}_{\eta}([0, \hat{S}])}{\hat{S}}-\mathbb{Q} \hat{S} e^{-\mathbb{Q}(\hat{S})^{\gamma}}>0
$$

where the last inequality follows by the fact that $\mathcal{H}_{\eta}([0, S])$ is increasing with respect to $S$. Hence,

$$
\lim _{S \rightarrow \infty} \frac{\mathcal{H}_{\eta}^{\Gamma}(x, S)}{S} \in(0, \infty)
$$

This completes the proof.

\subsection{Finite-time horizon. Let}

$$
\Delta_{1}(u)=\overleftarrow{\sigma}\left(\frac{\sqrt{2} \sigma^{2}(T)}{u+c T}\right), \quad \Delta_{2}(u)=\left(\frac{\sigma(T)}{u+c T}\right)^{2}
$$

Observe that for any $x \geq 0$

$$
\mathbb{P}\left(\frac{\mathcal{T}_{T}^{*}(u)-\tau_{T}^{*}(u)}{\Delta_{i}(u)}>x\right)=\frac{\mathbb{P}\left(\mathcal{T}_{T}(u)-\tau_{T}(u)>x \Delta_{i}(u), \tau_{T}(u) \leq T\right)}{\mathbb{P}\left(\tau_{T}(u) \leq T\right)}, \quad i=1,2 .
$$

In the following lemma we give exact asymptotics of $\mathbb{P}\left(\tau_{T}(u) \leq T\right)=\mathbb{P}\left(\sup _{t \in[0, T]} X(t)-c t>u\right)$, referring for the proof to Theorem 2.3 and Remark 2.4 in [7] (choose $\gamma=0)$.

Lemma 3.3. Let $X(t), t \in[0, T]$ be a centered Gaussian process with continuous trajectories and stationary increments satisfying BI-BII.

i) If $t=o\left(\sigma^{2}(t)\right), t \rightarrow 0$, then

$$
\mathbb{P}\left(\tau_{T}(u) \leq T\right) \sim \mathcal{H}_{B_{\alpha_{0}}}^{\Gamma}(0) \frac{\sigma^{3}(T)}{\dot{\sigma}(T)}\left(u^{2} \Delta_{1}(u)\right)^{-1} \Psi\left(\frac{u+c T}{\sigma(T)}\right) .
$$

ii) If $\sigma^{2}(t) \sim a t, t \rightarrow 0, a>0$, then for $d=\frac{2 \sigma(T) \dot{\sigma}(T)}{a}$,

$$
\mathbb{P}\left(\tau_{T}(u) \leq T\right) \sim \mathcal{P}_{B_{1 / 2}}^{d}(0) \Psi\left(\frac{u+c T}{\sigma(T)}\right)
$$

iii) If $\sigma^{2}(t)=o(t), t \rightarrow 0$, then

$$
\mathbb{P}\left(\tau_{T}(u) \leq T\right) \sim \Psi\left(\frac{u+c T}{\sigma(T)}\right) .
$$

Thus in order to derive the limit of (23) as $u \rightarrow \infty$, it suffices to find the asymptotics of

$$
\mathbb{P}\left(\mathcal{T}_{T}(u)-\tau_{T}(u)>x \Delta_{i}(u), \tau_{T}(u) \leq T\right), i=1,2, \quad u \rightarrow \infty
$$

Let

$$
\widetilde{\sigma}_{u}(t)=\frac{\sigma(t)}{u+c t} \frac{u+c T}{\sigma(T)}, 0 \leq t \leq T
$$


Lemma 3.4. Suppose that BI, BII hold. Then for u sufficiently large, $\widetilde{\sigma}_{u}(t)$ attains its maximum over $[0, T]$ at $T$, and for any $\delta_{u}>0$ with $\lim _{u \rightarrow \infty} \delta_{u}=0$

$$
\lim _{u \rightarrow \infty} \sup _{t \in\left[T-\delta_{u}, T\right]}\left|\frac{1-\widetilde{\sigma}_{u}(t)}{|t-T|}-\frac{\dot{\sigma}(T)}{\sigma(T)}\right|=0
$$

Moreover,

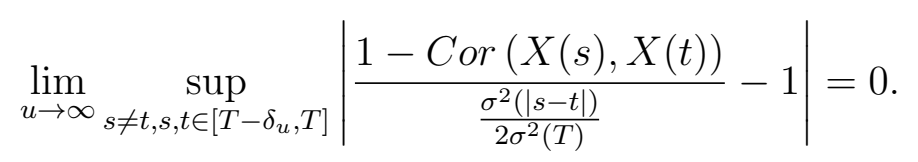

Proof of Theorem 2.3 By (23) and Lemma 3.3 it suffices to analyze asymptotics of

$$
\begin{aligned}
\mathbb{P}\left(\mathcal{T}_{T}(u)-\tau_{T}(u)>x \Delta_{j}(u), \tau_{T}(u) \leq T\right) \\
=\mathbb{P}\left(\mathcal{T}_{T}(u)-\tau_{T}(u)>x \Delta_{j}(u), \tau_{T}(u) \leq T, X(T)-C T<u\right) \\
\quad+\mathbb{P}\left(\mathcal{T}_{T}(u)-\tau_{T}(u)>x \Delta_{j}(u), \tau_{T}(u) \leq T, X(T)-C T>u\right) \\
=\mathbb{P}\left(\exists 0 \leq s, t \leq T, s-t \geq x \Delta_{j}(u), X(t)-c t>u, X(s)-c s>u, X(T)-c T<u\right) \\
\quad+\mathbb{P}\left(\exists 0 \leq t \leq T-x \Delta_{j}(u), X(t)-c t>u, X(T)-c T>u\right)
\end{aligned}
$$

where $j=1$ for cases i), ii) and $j=2$ for iii).

Thus, for $j=1,2$

$$
\sum_{i=3}^{4} \pi_{i}^{j}(u) \leq \mathbb{P}\left(\mathcal{T}_{T}(u)-\tau_{T}(u)>x \Delta_{j}(u), \tau_{T}(u) \leq T\right) \leq \sum_{i=3}^{4} \pi_{i}^{j}(u)+\pi_{5}(u),
$$

where

$$
\begin{aligned}
& \pi_{3}^{j}(u)=\mathbb{P}\left(\exists T-(\ln u / u)^{2} \leq s, t \leq T, s-t \geq x \Delta_{j}(u), X(t)-c t>u, X(s)-c s>u, X(T)-c T<u\right) \\
& \pi_{4}^{j}(u)=\mathbb{P}\left(\exists T-(\ln u / u)^{2} \leq t \leq T-x \Delta_{j}(u), X(t)-c t>u, X(T)-c T>u\right) \\
& \pi_{5}(u)=\mathbb{P}\left(\sup _{t \in\left[0, T-(\ln u / u)^{2}\right]} X(t)-c t>u\right) .
\end{aligned}
$$

Upper bound of $\pi_{5}(u)$. Rewrite $\pi_{5}(u)$ as

$$
\pi_{5}(u)=\mathbb{P}\left(\sup _{t \in\left[0, T-(\ln u / u)^{2}\right]} \frac{X(t)}{u+c t} \frac{u+c T}{\sigma(T)}>\frac{u+c T}{\sigma(T)}\right)
$$

By the stationarity of increments of $X$ and BII, we have

$$
\mathbb{E}\left\{\left(\frac{X(t)}{u+c t} \frac{u+c T}{\sigma(T)}-\frac{X(s)}{u+c s} \frac{u+c T}{\sigma(T)}\right)^{2}\right\} \leq \mathbb{Q}\left(|t-s|+\sigma^{2}(|t-s|)\right) \leq \mathbb{Q}|t-s|^{\min \left(\alpha_{0}, 1\right)}
$$

for $s, t \in[0, T]$. Moreover, following Lemma 3.4, we have that for $u$ sufficiently large

$$
\sup _{t \in\left[0, T-(\ln u / u)^{2}\right]} \operatorname{Var}\left(\frac{X(t)}{u+c t} \frac{u+c T}{\sigma(T)}\right) \leq 1-\mathbb{Q}_{1}(\ln u / u)^{2} .
$$

Consequently, by Piterbarg inequality (see, e.g., Theorem 8.1 in [27]), for $u$ sufficiently large

$$
\pi_{5}(u) \leq \mathbb{Q}_{2} u^{2 / \min \left(\alpha_{0}, 1\right)} \Psi\left(\frac{u+c T}{\sigma(T) \sqrt{1-\mathbb{Q}_{1}(\ln u / u)^{2}}}\right) .
$$


Asymptotics of $\pi_{i}^{j}(u), i=3,4, j=1,2$. Let

$$
X_{u}(t)=\frac{X(T-t)}{u+c(T-t)} \frac{u+c T}{\sigma(T)}, \quad 0 \leq t \leq T .
$$

Then for $j=1,2$

$$
\begin{aligned}
& \pi_{3}^{j}(u)=\mathbb{P}\left(\exists 0 \leq s, t \leq(\ln u / u)^{2}, t-s \geq x \Delta_{j}(u), X_{u}(t)>\frac{u+c T}{\sigma(T)}, X_{u}(s)>\frac{u+c T}{\sigma(T)}, X_{u}(0)<\frac{u+c T}{\sigma(T)}\right) \\
& \pi_{4}^{j}(u)=\mathbb{P}\left(\exists x \Delta_{j}(u) \leq t \leq(\ln u / u)^{2}, X_{u}(t)>\frac{u+c T}{\sigma(T)}, X_{u}(0)>\frac{u+c T}{\sigma(T)}\right) .
\end{aligned}
$$

In order to derive the asymptotics of $\pi_{i}^{j}(u), i=3,4, j=1,2$, we distinguish three scenarios: i) $t=o\left(\sigma^{2}(t)\right)$, ii) $\sigma^{2}(t) \sim$ at and iii) $\sigma^{2}(t)=o(t)$ as $t \rightarrow 0$.

$\diamond$ Case i) $t=o\left(\sigma^{2}(t)\right)$. Clearly,

$$
\pi_{4}^{1}(u) \leq \mathbb{P}\left(X_{u}(0)>\frac{u+c T}{\sigma(T)}\right)=\Psi\left(\frac{u+c T}{\sigma(T)}\right)
$$

We are left with deriving the asymptotics of $\pi_{3}^{1}(u)$.

Asymptotics of $\pi_{3}^{1}(u)$. We note that

$$
\hat{\pi}_{3}(u)-\mathbb{P}\left(X_{u}(0)>\frac{u+c T}{\sigma(T)}\right) \leq \pi_{3}^{1}(u) \leq \hat{\pi}_{3}(u)
$$

where

$$
\hat{\pi}_{3}(u)=\mathbb{P}\left(\exists 0 \leq s, t \leq(\ln u / u)^{2}, t-s \geq x \Delta_{1}(u), X_{u}(t)>\frac{u+c T}{\sigma(T)}, X_{u}(s)>\frac{u+c T}{\sigma(T)}\right) .
$$

For $S>x / 2$, let

$$
X_{u, k}(t)=X_{u}\left(\Delta_{1}(u)(k S+t)\right), \quad N_{1}(u)=\left[\frac{(\ln u)^{2}}{u^{2} \Delta_{1}(u) S}\right]-1 .
$$

Bonferroni inequality gives that

$$
\Sigma_{2}^{-}(u)-\Sigma \Sigma_{3}(u) \leq \hat{\pi}_{3}(u) \leq \Sigma_{2}^{+}(u),
$$

where

$$
\Sigma \Sigma_{3}(u)=\sum_{k=0}^{N_{1}(u)+1} \sum_{l=k+1}^{N_{1}(u)+1} \mathbb{P}\left(\sup _{t \in[k S,(k+1) S]} X_{u}\left(\Delta_{1}(u) t\right)>\frac{u+c T}{\sigma(T)}, \sup _{t \in[l S,(l+1) S]} X_{u}\left(\Delta_{1}(u) t\right)>\frac{u+c T}{\sigma(T)}\right),
$$

and

$$
\begin{aligned}
\Sigma_{2}^{ \pm}(u) & =\sum_{k=0}^{N_{1}(u) \pm 1} \mathbb{P}\left(\exists s \in[0, S], t \in[x+s, 2 S], X_{u, k}(t)>\frac{u+c T}{\sigma(T)}, X_{u, k}(s)>\frac{u+c T}{\sigma(T)}\right) \\
& =\sum_{k=0}^{N_{1}(u) \pm 1} \mathbb{P}\left(\sup _{t \in[0, S]} \min \left(X_{u, k}(t), \sup _{s \in[x+t, 2 S]} X_{u, k}(s)\right)>\frac{u+c T}{\sigma(T)}\right) \\
& =\sum_{k=0}^{N_{1}(u) \pm 1} \mathbb{P}\left(\Gamma\left(x, S ; X_{u, k}\right)>\frac{u+c T}{\sigma(T)}\right),
\end{aligned}
$$


with $\Gamma$ being defined in (3). By Lemma 3.4, we have that for any $0<\epsilon<1$

$$
\frac{\frac{u+c T}{\sigma(T)}}{\sup _{t \in[0,2 S]} \sqrt{\operatorname{Var}\left(X_{u, k}(t)\right)}}>\frac{u+c T}{\sigma(T)}\left(1+(1-\epsilon) \frac{\dot{\sigma}(T)}{\sigma(T)}|k| \Delta_{1}(u) S\right):=m_{k, \epsilon, 1}(u)
$$

as $u$ sufficiently large. This implies that

$$
\begin{aligned}
\mathbb{P}\left(\Gamma\left(x, S ; X_{u, k}\right)>\frac{u+c T}{\sigma(T)}\right) & \leq \mathbb{P}\left(\Gamma\left(x, S ; \bar{X}_{u, k}\right)>\frac{\frac{u+c T}{\sigma(T)}}{\sup _{t \in[0,2 S]} \sqrt{\operatorname{Var}\left(X_{u, k}(t)\right)}}\right) \\
& \leq \mathbb{P}\left(\Gamma\left(x, S ; \bar{X}_{u, k}\right)>m_{k, \epsilon, 1}(u)\right) .
\end{aligned}
$$

Moreover, by Lemma 3.4 we have

$$
\lim _{u \rightarrow \infty} \sup _{0 \leq k \leq N_{1}(u)+1} \sup _{s \neq t, s, t \in[0,2 S]}\left|m_{k, \epsilon, 1}^{2}(u) \frac{1-\operatorname{Cor}\left(X_{u, k}(t), X_{u, k}(s)\right)}{\frac{\sigma^{2}\left(\Delta_{1}(u)|t-s|\right)}{\sigma^{2}\left(\Delta_{1}(u)\right)}}-1\right|=0 .
$$

Thus, following Proposition 2.3 in [6], we have

$$
\sup _{|k| \leq N(u)+1}\left|\frac{\mathbb{P}\left(\Gamma\left(x, S ; \bar{X}_{u, k}(t)\right)>m_{k, \epsilon, 1}(u)\right)}{\Psi\left(m_{k, \epsilon, 1}(u)\right)}-\mathcal{H}_{B_{\alpha_{0}}}^{\Gamma}(x, S)\right|=0,
$$

which combined with (22) implies that

$$
\begin{aligned}
\Sigma_{2}^{+}(u) & \leq \sum_{k=0}^{N_{1}(u)+1} \mathcal{H}_{B_{\alpha_{0}}}^{\Gamma}(x, S) \Psi\left(m_{k, \epsilon, 1}(u)\right) \\
& \leq \mathcal{H}_{B_{\alpha_{0}}}^{\Gamma}(x, S) \Psi\left(\frac{u+c T}{\sigma(T)}\right) \sum_{k=0}^{N_{1}(u)+1} e^{-(1-\epsilon) \frac{\dot{\sigma}(T)}{\sigma^{3}(T)}|k| u^{2} \Delta_{1}(u) S} \\
& \leq \frac{\mathcal{H}_{B_{\alpha_{0}}}^{\Gamma}(x, S)}{S} \frac{\sigma^{3}(T)}{(1-\epsilon) \dot{\sigma}(T) u^{2} \Delta_{1}(u)} \Psi\left(\frac{u+c T}{\sigma(T)}\right) \int_{0}^{\infty} e^{-t} d t \\
& \sim \mathcal{H}_{B_{\alpha_{0}}}^{\Gamma}(x) \Theta_{1}(u), \quad u \rightarrow \infty, S \rightarrow \infty, \epsilon \rightarrow 0,
\end{aligned}
$$

with

Analogously,

$$
\Theta_{1}(u)=\frac{\sigma^{3}(T)}{\dot{\sigma}(T)}\left(u^{2} \Delta_{1}(u)\right)^{-1} \Psi\left(\frac{u+c T}{\sigma(T)}\right)
$$

$$
\Sigma_{2}^{-}(u) \geq \mathcal{H}_{B_{\alpha_{0}}}^{\Gamma}(x) \Theta_{1}(u)(1+o(1)), \quad u \rightarrow \infty, S \rightarrow \infty .
$$

Following similar arguments as in (18)-(19), substituting $\eta$ by $B_{\alpha_{0}}$ and $\Theta(u)$ by $\Theta_{1}(u)$ in (18)-(19), we derive that

$$
\begin{aligned}
\Sigma \Sigma_{3}(u) & \leq\left(2 \frac{\mathcal{H}_{B_{\alpha_{0}}}([0, S])}{S}-\frac{\mathcal{H}_{B_{\alpha_{0}}}([0,2 S])}{S}+\mathbb{Q} S e^{-\mathbb{Q} S^{\gamma}}\right) \Theta_{1}(u) \\
& =o\left(\Theta_{1}(u)\right), \quad u \rightarrow \infty, S \rightarrow \infty .
\end{aligned}
$$

Therefore,

$$
\hat{\pi}_{3}(u) \sim \mathcal{H}_{B_{\alpha_{0}}}^{\Gamma}(x) \Theta_{1}(u), \quad u \rightarrow \infty
$$

By the fact that

$$
\hat{\pi}_{3}(u)-\mathbb{P}\left(X_{u}(0)>\frac{u+c T}{\sigma(T)}\right) \leq \pi_{3}^{1}(u) \leq \hat{\pi}_{3}(u)
$$


we have

$$
\pi_{3}^{1}(u) \sim \mathcal{H}_{B_{\alpha_{0}}}^{\Gamma}(x) \Theta_{1}(u), \quad u \rightarrow \infty
$$

which combined with (24)-(26) leads to

$$
\mathbb{P}\left(\mathcal{T}_{T}(u)-\tau_{T}(u)>x \Delta_{j}(u), \tau_{T}(u) \leq T\right) \sim \mathcal{H}_{B_{\alpha_{0}}}^{\Gamma}(x) \Theta_{1}(u), \quad u \rightarrow \infty
$$

Inserting the above and i) in Lemma 3.3 to (23), we establish the claim.

$\diamond$ ii) Case $\sigma^{2}(t) \sim$ at. In this case we choose $\Delta_{1}(u)=\overleftarrow{\sigma}\left(\frac{\sqrt{2} \sigma^{2}(T)}{u+c T}\right)$ as the scaling function. Asymptotics of $\pi_{3}^{1}(u)$. Using notation introduced in (27), we have for $S>x$,

$$
\pi_{6}(u) \leq \pi_{3}^{1}(u) \leq \pi_{6}(u)+\Sigma_{3}(u)
$$

where

$$
\begin{aligned}
\pi_{6}(u) & =\mathbb{P}\left(\exists 0 \leq s \leq s+x \leq t \leq S, X_{u, 0}(t)>\frac{u+c T}{\sigma(T)}, X_{u, 0}(s)>\frac{u+c T}{\sigma(T)}, X_{u, 0}(0)<\frac{u+c T}{\sigma(T)}\right) \\
& =\mathbb{P}\left(\Gamma^{\prime}\left(x, S ; X_{u, 0}\right)>\frac{u+c T}{\sigma(T)}, X_{u, 0}(0)<\frac{u+c T}{\sigma(T)}\right)
\end{aligned}
$$

with

$$
\Gamma^{\prime}(x, S ; f):=\sup _{t \in[x, S]} \min \left(f(t), \sup _{s \in[0, t-x]} f(s)\right)
$$

and

$$
\Sigma_{3}(u)=\sum_{k=1}^{N_{1}(u)+1} \mathbb{P}\left(\sup _{t \in[0, S]} X_{u, k}(t)>\frac{u+c T}{\sigma(T)}\right)
$$

Asymptotics of $\pi_{6}(u)$. We begin with observation that

$$
\pi_{6}(u)=\mathbb{P}\left(\Gamma^{\prime}\left(x, S ; \frac{\bar{X}_{u, 0}(t)}{1+\left(1 / \sqrt{\operatorname{Var}\left(X_{u, 0}(t)\right)}-1\right)}\right)>\frac{u+c T}{\sigma(T)}, X_{u, 0}(0)<\frac{u+c T}{\sigma(T)}\right) .
$$

We shall apply Lemma 3.5 from Appendix, for which we verify assumptions D0-D2 (see Appendix). Note that D0 holds straightforwardly. From (28) for $k=0$, we know that D1 is satisfied. By Lemma 3.4 , it follows that

$$
\lim _{u \rightarrow \infty} \sup _{t \in[0, S]}\left|\left(\frac{u+c T}{\sigma(T)}\right)^{2}\left(1 / \sqrt{\operatorname{Var}\left(X_{u, 0}(t)\right)}-1\right)-\frac{2 \sigma(T) \dot{\sigma}(T)}{a} t\right|=0 .
$$

This implies that D2 is satisfied with $h(t)=\frac{2 \sigma(T) \dot{\sigma}(T)}{a} t$. Moreover,

$$
\left\{X_{u, 0}(0)<\frac{u+c T}{\sigma(T)}\right\}=\left\{X_{u, 0}(0)=\frac{u+c T}{\sigma(T)}-\frac{w}{\frac{u+c T}{\sigma(T)}}, w \in D\right\}
$$

with $D=(0, \infty)$. Thus

$$
\pi_{6}(u) \sim \int_{0}^{\infty} e^{w} \mathbb{P}\left(\Gamma^{\prime}(x, S ; W)>w\right) d w \Psi\left(\frac{u+c T}{\sigma(T)}\right), \quad u \rightarrow \infty .
$$

with

$$
W(t)=\sqrt{2} B_{1 / 2}(t)-\left(1+\frac{2 \sigma(T) \dot{\sigma}(T)}{a}\right) t
$$


Upper bound for $\Sigma_{3}(u)$. Noting that $\Gamma(0, S ; f)=\sup _{t \in[0, S]} f(t)$, by $(29)$ we have

$$
\begin{aligned}
\Sigma_{3}(u) & \leq \sum_{k=1}^{N_{1}(u)+1} \mathbb{P}\left(\sup _{t \in[0, S]} \bar{X}_{u, k}(t)>m_{k, \epsilon, 1}(u)\right) \\
& \leq \sum_{k=1}^{N_{1}(u)+1} \mathcal{H}_{B_{1 / 2}}([0, S]) \Psi\left(m_{k, \epsilon, 1}(u)\right) \\
& \leq \mathcal{H}_{B_{1 / 2}}([0, S]) \Psi\left(\frac{u+c T}{\sigma(T)}\right) \sum_{k=1}^{N_{1}(u)+1} e^{-(1-\epsilon) \frac{\dot{\sigma}(T)}{\sigma^{3}(T)} k u^{2} \Delta_{1}(u) S} \\
& \leq \mathbb{Q} S \sum_{k=1}^{N_{1}(u)+1} e^{-\mathbb{Q}_{1} k S} \Psi\left(\frac{u+c T}{\sigma(T)}\right) \\
& \leq \mathbb{Q} S e^{-\mathbb{Q}_{2} S} \Psi\left(\frac{u+c T}{\sigma(T)}\right)=o\left(\Psi\left(\frac{u+c T}{\sigma(T)}\right)\right), \quad u \rightarrow \infty, S \rightarrow \infty
\end{aligned}
$$

Therefore,

$$
\pi_{3}^{1}(u) \sim \int_{0}^{\infty} e^{w} \mathbb{P}\left(\sup _{t \in[x, \infty)} \min \left(\sup _{s \in[0, t-x]} \eta(s), \eta(t)\right)>w\right) d w \Psi\left(\frac{u+c T}{\sigma(T)}\right), \quad u \rightarrow \infty
$$

Asymptotics of $\pi_{4}^{1}(u)$. Observe that

$$
\pi_{7}(u) \leq \pi_{4}^{1}(u) \leq \pi_{7}(u)+\Sigma_{3}(u),
$$

where $\Sigma_{3}(u)$ is given by $(30)$ and

$$
\begin{aligned}
\pi_{7}(u) & =\mathbb{P}\left(\exists x \leq t \leq S, X_{u, 0}(t)>\frac{u+c T}{\sigma(T)}, X_{u, 0}(0)>\frac{u+c T}{\sigma(T)}\right) \\
& =\mathbb{P}\left(\sup _{t \in[x, S]} X_{u, 0}(t)>\frac{u+c T}{\sigma(T)}, X_{u, 0}(0)>\frac{u+c T}{\sigma(T)}\right) .
\end{aligned}
$$

Note that

$$
\left\{X_{u, 0}(0)>\frac{u+c T}{\sigma(T)}\right\}=\left\{X_{u, 0}(0)=\frac{u+c T}{\sigma(T)}-\frac{w}{\frac{u+c T}{\sigma(T)}}, w \in D\right\}
$$

with $D=(-\infty, 0)$. By (28), (31) and (32), applying Remark 3.6 in Appendix, it follows that

$$
\pi_{7}(u) \sim \int_{-\infty}^{0} e^{w} \mathbb{P}\left(\sup _{t \in[x, S]} W(t)>w\right) d w \Psi\left(\frac{u+c T}{\sigma(T)}\right),
$$

with $W$ given in (33). Inserting the above asymptotics and (34) into (26) gives that

$$
\pi_{4}^{1}(u) \sim \int_{-\infty}^{0} e^{w} \mathbb{P}\left(\sup _{t \in[x, S]} W(t)>w\right) d w \Psi\left(\frac{u+c T}{\sigma(T)}\right), \quad u \rightarrow \infty .
$$

Combination of (24), (25), (35) and (37) gives the asymptotics for $\mathbb{P}\left(\mathcal{T}_{T}(u)-\tau_{T}(u)>x \Delta_{1}(u), \tau_{T}(u) \leq T\right)$, which together with ii) in Lemma 3.3 establishes the claim.

$\diamond$ Case iii) $\sigma^{2}(t)=o(t)$. In this case we choose $\Delta_{2}(u)=\left(\frac{\sigma(T)}{u+c T}\right)^{2}$ as the scaling function. Asymptotics of $\pi_{4}^{2}(u)$. Observe that

$$
\pi_{8}(u) \leq \pi_{4}^{2}(u) \leq \pi_{8}(u)+\Sigma_{4}(u),
$$


where

$$
\begin{aligned}
\pi_{8}(u) & =\mathbb{P}\left(\exists x \leq t \leq S, \widehat{X}_{u, 0}(t)>\frac{u+c T}{\sigma(T)}, \widehat{X}_{u, 0}(0)>\frac{u+c T}{\sigma(T)}\right) \\
& =\mathbb{P}\left(\sup _{t \in[x, S]} \widehat{X}_{u, 0}(t)>\frac{u+c T}{\sigma(T)}, \widehat{X}_{u, 0}(0)>\frac{u+c T}{\sigma(T)}\right) \\
& =\mathbb{P}\left(\sup _{t \in[x, S]} \frac{\bar{X}_{u, 0}(t)}{1+1 / \sqrt{\operatorname{Var}\left(\widehat{X}_{u, 0}(t)\right)}-1}>\frac{u+c T}{\sigma(T)}, \widehat{X}_{u, 0}(0)>\frac{u+c T}{\sigma(T)}\right) \\
\Sigma_{4}(u) & =\sum_{k=1}^{N_{2}(u)+1} \mathbb{P}\left(\sup _{t \in[0, S]} \widehat{X}_{u, k}(t)>\frac{u+c T}{\sigma(T)}\right) \\
& \leq \sum_{k=1}^{N_{2}(u)+1} \mathbb{P}\left(\sup _{t \in[0, S]} \widehat{\widehat{X}}_{u, k}(t)>m_{k, \epsilon, 2}(u)\right)
\end{aligned}
$$

where

$$
\begin{gathered}
\widehat{X}_{u, k}(t)=X_{u}\left(\Delta_{2}(u)(k S+t)\right), \quad N_{2}(u)=\left[\frac{(\ln u)^{2}}{u^{2} \Delta_{2}(u) S}\right], \\
m_{k, \epsilon, 2}(u)=\frac{u+c T}{\sigma(T)}\left(1+(1-\epsilon) \frac{\dot{\sigma}(T)}{\sigma(T)}|k| \Delta_{2}(u) S\right) .
\end{gathered}
$$

Asymptotics of $\pi_{8}(u)$. In order to apply Lemma 3.5 and Remark 3.6 in Appendix, we check D0-D2. Note that Do hold straightforwardly. By Lemma 3.4, it follows that

$$
\lim _{u \rightarrow \infty} \sup _{|k| \leq N_{2}(u)+1} \sup _{s \neq t, s, t \in[0, S]}\left|\left(m_{k, \epsilon, 2}(u)\right)^{2} \frac{1-\operatorname{Cor}\left(\widehat{X}_{u, k}(s), \widehat{X}_{u, k}(t)\right.}{\frac{\sigma^{2}\left(\Delta_{2}(u)|t-s|\right)}{\sigma^{2}\left(\Delta_{1}(u)\right)}}-1\right|=0
$$

with

$$
\lim _{u \rightarrow \infty} \frac{\Delta_{2}(u)}{\Delta_{1}(u)}=0
$$

This implies that $\mathbf{D} 1$ holds with $\nu=0$. Lemma 3.4 indicates that

$$
\lim _{u \rightarrow \infty} \sup _{t \in[0, S]}\left|\left(\frac{u+c T}{\sigma(T)}\right)^{2}\left(1 / \sqrt{\operatorname{Var}\left(\widehat{X}_{u, 0}(t)\right)-1}\right)-\frac{\dot{\sigma}(T)}{\sigma(T)} t\right|=0 .
$$

This means that D2 holds with $h(t)=\frac{\dot{\sigma}(T)}{\sigma(T)} t$. Moreover,

$$
\left\{\widehat{X}_{u, 0}(0)>\frac{u+c T}{\sigma(T)}\right\}=\left\{\widehat{X}_{u, 0}(0)=\frac{u+c T}{\sigma(T)}-\frac{w}{\frac{u+c T}{\sigma(T)}}, w \in D\right\},
$$

with $D=(-\infty, 0)$. Hence

$$
\begin{aligned}
\pi_{8}(u) & \sim \int_{-\infty}^{0} e^{w} \mathbb{P}\left(\sup _{t \in[x, S]}-\frac{\dot{\sigma}(T)}{\sigma(T)} t>w\right) d w \Psi\left(\frac{u+c T}{\sigma(T)}\right) \\
& =e^{-\frac{\dot{\sigma}(T)}{\sigma(T)} x} \Psi\left(\frac{u+c T}{\sigma(T)}\right)
\end{aligned}
$$


Upper bound of $\Sigma_{4}(u)$. Similarly, for the summands in $\Sigma_{4}(u)$ we can show that D0-D2 hold with $h(t)=\frac{\dot{\sigma}(T)}{\sigma(T)} t$ and $\nu=0$. Thus by Remark 3.6 in Appendix, we have

$$
\lim _{u \rightarrow \infty} \sup _{|k| \leq N_{2}(u)+1}\left|\frac{\mathbb{P}\left(\sup _{t \in[0, S]} \widehat{\widehat{X}}_{u, k}(t)>m_{k, \epsilon, 2}(u)\right)}{\Psi\left(m_{k, \epsilon, 2}(u)\right)}-\int_{-\infty}^{\infty} e^{w} \mathbb{P}\left(\sup _{t \in[0, S]}-\frac{\dot{\sigma}(T)}{\sigma(T)} t>w\right) d w\right|=0,
$$

where

$$
\int_{-\infty}^{\infty} e^{w} \mathbb{P}\left(\sup _{t \in[0, S]}-\frac{\dot{\sigma}(T)}{\sigma(T)} t>w\right) d w=1
$$

Hence

$$
\begin{aligned}
\Sigma_{4}(u) & \leq \sum_{k=1}^{N_{2}(u)+1} \Psi\left(m_{k, \epsilon, 2}(u)\right) \\
& \leq \Psi\left(\frac{u+c T}{\sigma(T)}\right) \sum_{k=1}^{N_{2}(u)+1} e^{-\mathbb{Q}|k| \Delta_{2}(u) u^{2} S} \\
& \leq \Psi\left(\frac{u+c T}{\sigma(T)}\right) \sum_{k=1}^{N_{2}(u)+1} e^{-\mathbb{Q}_{1} S} \\
& \leq e^{-\mathbb{Q}_{2} S} \Psi\left(\frac{u+c T}{\sigma(T)}\right)=o\left(\Psi\left(\frac{u+c T}{\sigma(T)}\right)\right), \quad u \rightarrow \infty, S \rightarrow \infty
\end{aligned}
$$

Thus

$$
\pi_{4}^{2}(u) \sim e^{-\frac{\dot{\sigma}(T)}{\sigma(T)} x} \Psi\left(\frac{u+c T}{\sigma(T)}\right), \quad u \rightarrow \infty .
$$

Upper bound of $\pi_{3}^{2}(u)$. It follows that

$$
\begin{aligned}
\pi_{3}^{2}(u) & \leq \mathbb{P}\left(\tau_{T}(u) \leq T, X_{u}(0)<\frac{u+c T}{\sigma(T)}\right) \\
& =\mathbb{P}\left(\tau_{T}(u) \leq T\right)-\mathbb{P}\left(\tau_{T}(u) \leq T, X_{u}(0)>\frac{u+c T}{\sigma(T)}\right) \\
& =\mathbb{P}\left(\tau_{T}(u) \leq T\right)-\mathbb{P}\left(X_{u}(0)>\frac{u+c T}{\sigma(T)}\right)
\end{aligned}
$$

Applying iii) in Lemma 3.3, we have

$$
\pi_{3}^{2}(u)=o\left(\Psi\left(\frac{u+c T}{\sigma(T)}\right)\right), \quad u \rightarrow \infty .
$$

Recalling (24)-(25), we conclude that

$$
\mathbb{P}\left(\mathcal{T}_{T}(u)-\tau_{T}(u)>x \Delta_{2}(u), \tau_{T}(u) \leq T\right) \sim e^{-\frac{\dot{\sigma}(T)}{\sigma(T)} x} \Psi\left(\frac{u+c T}{\sigma(T)}\right), \quad u \rightarrow \infty,
$$

which combined with (23) and iii) in Lemma 3.3 leads, for any $x>0$, to

$$
\lim _{u \rightarrow \infty} \mathbb{P}\left(\frac{\mathcal{T}_{T}^{*}(u)-\tau_{T}^{*}(u)}{\Delta_{2}(u)}>x\right)=e^{-\frac{\dot{\sigma}(T)}{\sigma(T)} x} .
$$

This completes the proof. 


\section{APPENDIX}

In this section we give a variant of Theorem 2.1 in [6]. Let $\xi_{u, \tau_{u}}$ be a family of Gaussian random fields given by

$$
\xi_{u, \tau_{u}}(t)=\frac{Z_{u, \tau_{u}}(t)}{1+h_{u, \tau_{u}}(t)}, \quad t \in[0, S], \tau_{u} \in K_{u}
$$

where $Z_{u, \tau_{u}}$ is a family of centered Gaussian random fields with continuous trajectories and unit variance, $h_{u, \tau_{u}} \in C([0, S]), S>0$ and $K_{u}$ is a set of index. We investigate the asymptotics of

$$
\mathbb{P}\left(\Gamma^{\prime}\left(x, S ; \xi_{u, \tau_{u}}\right)>g_{u, \tau_{u}}, D_{u, \tau_{u}}\right)
$$

as $u \rightarrow \infty$ where $g_{u, \tau_{u}}$ is a series of positive functions of $u$,

$$
D_{u, \tau_{u}}=\left\{Z_{u, \tau_{u}}(0)=g_{u, \tau_{u}}-\frac{w}{g_{u, \tau_{u}}}, w \in D\right\}
$$

with $D=(0, \infty),(-\infty, 0)$ or $D=\mathbb{R}$ and $\Gamma^{\prime}: C([0, S]) \rightarrow \mathbb{R}, 0 \leq x \leq S$ is a real-valued continuous functional defined by

$$
\Gamma^{\prime}(x, S ; f)=\sup _{t \in[x, S]} \min \left(f(t), \sup _{s \in[0, t-x]} f(s)\right), \quad f \in C([0, S]) .
$$

In order to avoid trivialities, we assume that

$$
\lim _{u \rightarrow \infty} \mathbb{P}\left(\Gamma^{\prime}\left(x, S ; \xi_{u, \tau_{u}}\right)>g_{u, \tau_{u}}, D_{u, \tau_{u}}\right)=0
$$

and observe that $\mathbb{P}\left(\Gamma^{\prime}\left(x, S ; \xi_{u, \tau_{u}}\right)>g_{u, \tau_{u}}, D_{u, \tau_{u}}\right)=\mathbb{P}\left(\Gamma^{\prime}\left(x, S ; \xi_{u, \tau_{u}}\right)>g_{u, \tau_{u}}\right)$ if $D=\mathbb{R}$.

As in [6] (see Theorem 2.1), we impose the following assumptions:

D0: $\lim _{u \rightarrow \infty} \inf _{\tau_{u} \in K_{u}} g_{u, \tau_{u}}=\infty$.

D1: There exist $\rho(t)$, regularly varying function at 0 with index $2 \alpha_{0} \in(0,2]$ and $b_{i}(u)>0, i=1,2$ satisfying $\lim _{u \rightarrow \infty} b_{i}(u)=0, i=1,2$ and $\lim _{u \rightarrow \infty} \frac{b_{1}(u)}{b_{2}(u)}=\nu \in[0, \infty)$ such that

$$
\lim _{u \rightarrow \infty} \sup _{\tau_{u} \in K_{u}} \sup _{s, t \in[0, S], s \neq t}\left|\left(g_{u, \tau_{u}}\right)^{2} \frac{1-\operatorname{Corr}\left(Z_{u, \tau_{u}}(t), Z_{u, \tau_{u}}(s)\right)}{\frac{\rho\left(b_{1}(u)|t-s|\right)}{\rho\left(b_{2}(u)\right)}-1}\right|=0 .
$$

D2: There exists $h \in C([0, S])$ such that

$$
\lim _{u \rightarrow \infty} \sup _{\tau_{u} \in K_{u}} \sup _{t \in[0, S]}\left|\left(g_{u, \tau_{u}}\right)^{2} h_{u, \tau_{u}}(t)-h(t)\right|=0 .
$$

Lemma 3.5. Let $\xi_{u, \tau_{u}}$ be defined as in (38) and $\Gamma^{\prime}$ be defined in (40). Assume that D0-D2 are satisfied. Then, for $D_{u}, \tau_{u}$ defined in (39) with $D=(0, \infty),(-\infty, 0)$ or $D=\mathbb{R}$,

$\lim _{u \rightarrow \infty} \sup _{\tau_{u} \in K_{u}}\left|\frac{\mathbb{P}\left(\Gamma^{\prime}\left(x, S ; \xi_{u, \tau_{u}}\right)>g_{u, \tau_{u}}, D_{u, \tau_{u}}\right)}{\Psi\left(g_{u, \tau_{u}}\right)}-\int_{D} e^{w} \mathbb{P}\left(\Gamma^{\prime}\left(x, S ; \nu^{\alpha_{0}} B_{\alpha_{0}}(t)-\nu^{2 \alpha_{0}}|t|^{2 \alpha_{0}}-h(t)\right)>w\right) d w\right|=0$.

Proof. Conditioning on the event that $Z_{u, \tau_{u}}(0)=g_{u, \tau_{u}}-\frac{w}{g_{u, \tau_{u}}}$ and noting that $D_{u, \tau_{u}}=\left\{Z_{u, \tau_{u}}(0)=\right.$ $\left.g_{u, \tau_{u}}-\frac{w}{g_{u, \tau_{u}}}, w \in D\right\}$, we have

$$
\begin{aligned}
& \mathbb{P}\left(\Gamma^{\prime}\left(x, S ; \xi_{u, \tau_{u}}\right)>g_{u, \tau_{u}}, D_{u, \tau_{u}}\right) \\
& =\frac{1}{\sqrt{2 \pi} g_{u, \tau_{u}}} \int_{\mathbb{R}} e^{-\frac{\left(g_{u, \tau_{u}}-\frac{w}{g_{u, \tau_{u}}}\right)^{2}}{2}} \mathbb{P}\left(\Gamma^{\prime}\left(x, S ; \xi_{u, \tau_{u}}\right)>g_{u, \tau_{u}}, D_{u, \tau_{u}} \mid Z_{u, \tau_{u}}(0)=g_{u, \tau_{u}}-\frac{w}{g_{u, \tau_{u}}}\right) d w
\end{aligned}
$$




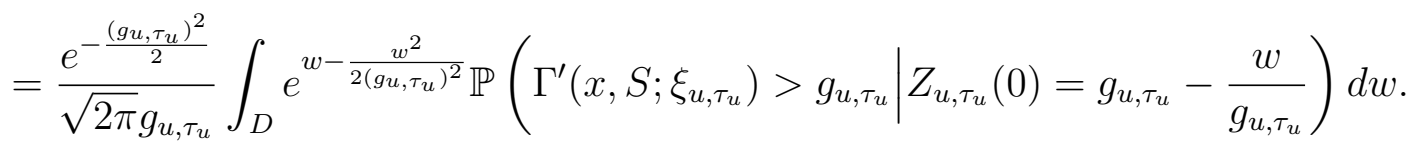

Using the same procedure as in the proof of Theorem 2.1 in [6], we can show that

$$
\int_{D} e^{w-\frac{w^{2}}{2\left(g_{u}, \tau_{u}\right)^{2}}} \mathbb{P}\left(\Gamma^{\prime}\left(x, S ; \xi_{u, \tau_{u}}\right)>g_{u, \tau_{u}} \mid Z_{u, \tau_{u}}(0)=g_{u, \tau_{u}}-\frac{w}{g_{u, \tau_{u}}}\right) d w
$$

uniformly converges to

$$
\int_{D} e^{w} \mathbb{P}\left(\Gamma^{\prime}\left(x, S ; \nu^{\alpha_{0}} B_{\alpha_{0}}(t)-\nu^{2 \alpha_{0}}|t|^{2 \alpha_{0}}-h(t)\right)>w\right) d w
$$

with respect to $\tau_{u} \in K_{u}$. This completes the proof.

Remark 3.6. Lemma 3.5 also holds if we substitute $\Gamma^{\prime}$ by $\sup _{t \in[x, S]} f(t)$, with $f \in C[x, S], x \geq 0$ and $D$, a measurable subset of $\mathbb{R}$ with positive Lebesgue measure.

Acknowledgement: We thank Enkelejd Hashorva for discussions and comments that improved presentation of the results of this contribution. K. Dȩbicki was partially supported by NCN Grant No 2015/17/B/ST1/01102 (2016-2019) whereas P. Liu was supported by the Swiss National Science Foundation Grant 200021-175752/1.

\section{REFERENCES}

[1] L. Bai, K. Dȩbicki, E. Hashorva, and L. Luo. On generalised Piterbarg constants. Comp. Meth. Appl. Prob., doi:10.100\%/s11009-016-9537-0, 2017.

[2] M. Chesney, M. Jeanblanc-Picqué, and M. Yor. Brownian excursions and Parisian barrier options. Adv. in Appl. Probab., 29(1):165-184, 1997.

[3] S. Chiu and C. Yin. Passage times for a spectrally negative Lévy process with applications to risk theory. Bernoulli, 11(3):511-522, 2005.

[4] K. Dębicki, E. Hashorva, and L. Ji. Parisian ruin of self-similar Gaussian risk processes. J. Appl. Probab., 52(3):688-702, 2015.

[5] K. Dębicki, E. Hashorva, and L. Ji. Parisian ruin over a finite-time horizon. Sci. China Math., $59(3): 557-572,2016$.

[6] K. Dębicki, E. Hashorva, and P. Liu. Uniform tail approximation of homogenous functionals of Gaussian fields. Adv. in Appl. Probab., 49:1037-1066, 2017.

[7] K. Dębicki, E. Hashorva, and P. Liu. Extremes of $\gamma$-reflected Gaussian process with stationary increments. ESAIM Probab. Statist., doi:10.1051/ps/2017019, 2017.

[8] K. Dȩbicki and K. Kosiński. On the infimum attained by the reflected fractional Brownian motion. Extremes, 17(3):431-446, 2014.

[9] K. Dȩbicki and P. Liu. Extremes of stationary Gaussian storage models. Extremes, 19(2):273302, 2016.

[10] K. Dȩbicki. Ruin probability for Gaussian integrated processes. Stochastic Process. Appl., 98(1):151-174, 2002. 
[11] A. B. Dieker. Extremes of Gaussian processes over an infinite horizon. Stochastic Process. Appl., 115(2):207-248, 2005.

[12] A. B. Dieker and T. Mikosch. Exact simulation of Brown-Resnick random fields at a finite number of locations. Extremes, 18:301-314, 2015.

[13] A. B. Dieker and B. Yakir. On asymptotic constants in the theory of Gaussian processes. Bernoulli, 20(3):1600-1619, 2014.

[14] E. Frostig. Upper bounds on the expected time to ruin and on the expected recovery time. Adv. in Appl. Probab., 36(2):377-397, 2004.

[15] H. Guérin and J.-F. Renaud. On the distribution of cumulative Parisian ruin. Insurance Math. Econom., 73:116-123, 2017.

[16] A. J. Harper. Bounds on the suprema of Gaussian processes, and omega results for the sum of a random multiplicative function. Ann. Appl. Probab, 23:584-616, 2013.

[17] A.J. Harper. Pickands' constant $H_{\alpha}$ does not equal $1 / \Gamma(1 / \alpha)$, for small $\alpha$. Bernoulli, 23(1):582$602,2017$.

[18] E. Hashorva and L. Ji. Approximation of passage times of $\gamma$-reflected processes with fBm input. J. Appl. Probab., 51(3):713-726, 2014.

[19] J. Hüsler and V. I. Piterbarg. On the ruin probability for physical fractional Brownian motion. Stochastic Process. Appl., 113(2):315-332, 2004.

[20] J. Hüsler and V.I. Piterbarg. A limit theorem for the time of ruin in a Gaussian ruin problem. Stochastic Process. Appl., 118(11):2014-2021, 2008.

[21] J. Hüsler and Y. Zhang. On first and last ruin times of Gaussian processes. Statist. Probab. Lett., 78(10):1230-1235, 2008.

[22] D.L. Iglehart. Diffusion approximations in collective risk theory. J. Appl. Probability, 6:285-292, 1969.

[23] S. Li. The time of recovery and the maximum severity of ruin in a Sparre Andersen model. $N$. Am. Actuar. J., 12(4):413-427, 2008.

[24] P. Liu, E. Hashorva, and L. Ji. On the $\gamma$-reflected processes with fBm input. Lithuanian Math J., 55(3):402-412, 2015.

[25] R. Loeffen, I. Czarna, and Z. Palmowski. Parisian ruin probability for spectrally negative Lévy processes. Bernoulli, 19(2):599-609, 2013.

[26] Z. Michna. Self-similar processes in collective risk theory. J. Appl. Math. Stochastic Anal., 11(4):429-448, 1998.

[27] V. I. Piterbarg. Asymptotic methods in the theory of Gaussian processes and fields, volume 148 of Translations of Mathematical Monographs. American Mathematical Society, Providence, RI, 1996. 
Krzysztof Dȩbicki, Mathematical Institute, University of WrocŁaW, Pl. Grunwaldzki 2/4, 50-384 Wroceaw, Poland

E-mail address: Krzysztof.Debicki@math.uni.wroc.pl

Peng liu, Department of Actuarial Science, Faculty of Business and Economics, University of Lausanne, UnIL-Dorigny 1015 Lausanne, Switzerland

E-mail address: peng.liu@unil.ch 